\title{
Ultrasonic Characterization of Interfaces in Composite Bonds
}

\author{
N. Wang ${ }^{1}$, O. I. Lobkis ${ }^{1}$, S. I. Rokhlin ${ }^{1}$, and J. H. Cantrell ${ }^{2}$ \\ 1 The Ohio State University, Edison Joining Technology Center, 1248 Arthur E Adams Drive, \\ Columbus $\mathrm{OH} 43221$ \\ 2 NASA Langley Research Center, Mail Stop 231, Hampton, Virginia 23681
}

\begin{abstract}
The inverse determination of imperfect interfaces from reflection spectra of normal and oblique incident ultrasonic waves in adhesive bonds of multidirectional composites is investigated. The oblique measurements are complicated by the highly dispersed nature of oblique wave spectra at frequencies above $3 \mathrm{MHz}$. Different strategies for bond property reconstruction, including a modulation method, are discussed. The relation of measured interfacial spring density to the physico-chemical model of a composite interface described by polymer molecular bonds to emulate loss of molecular strength on an adhesive composite interface is discussed. This potentially relates the interfacial (adhesion) strength (number of bonds at the adhesive substrate interface) to the spring constant (stiffness) area density (flux), which is an ultrasonically measurable parameter.
\end{abstract}

Keywords: ultrasonic, composite bonds, interface, molecular bonds

PACS: 43.35.Zc, 68.35.Iv, 68.35.Np, 81.70.Cv, 82.35.Gh

\section{INTRODUCTION}

Adhesive bonding of composite has found wide application in the aerospace industry because of the versatility of the process to accommodate design needs. However, due to possible surface preparation anomalies in manufacturing processes an adhesive bond may sufferer unexpected strength reduction and/or be susceptible to environmental degradation of bonding interfaces in service. Possible mechanisms of the environmental interface degradation may include distributed loss of molecular bonds leading eventually to micro-disbonds, interfacial strength reduction and failure.

Interfacial degradation of adhesive bonds is difficult to detect by standard ultrasonic inspection methods especially at initial degradation stages. To mitigate these difficulties, linear [1] and modulated [2] angle beam ultrasonic spectroscopy (ABUS) methods have been developed to determine properties and quality of adhesive joints. The application of these methods for characterization of environmental degradation of adhesive bonds of aluminum has been well documented $[2,3]$. In this paper, current progress and improvement of these two experimental methodologies are discussed with a focus on composite bond applications. In addition, the statistical physico-chemical model is described relating ultrasonic characteristics to bond property quantities: fraction of molecular bond loss and fraction of disbonded area. 
a)

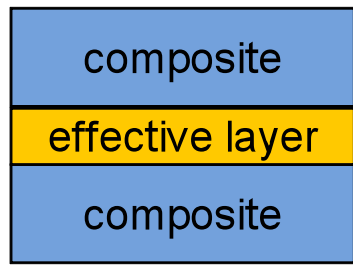

Effectivelayer properties

$\overline{\lambda+2 \mu}, \bar{\mu}, \bar{\rho}, \bar{h}, \overline{\alpha_{l}}, \overline{\alpha_{t}}$ b)

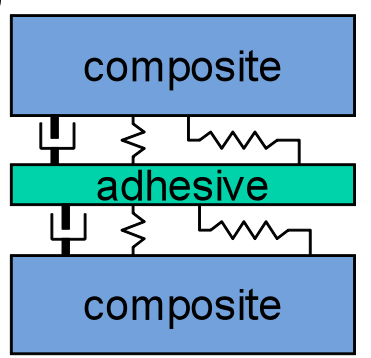

Indep end ent interf a cialproperties

$K_{n}=K_{n}^{\prime}+i K_{n}^{\prime \prime}, K_{t}=K_{t}^{\prime}+i K_{t}^{\prime \prime}$

Ind ep endent adhesive properties

$\lambda+2 \mu, \mu, \rho, h, \alpha_{l}, \alpha_{t}$

FIGURE 1. a) Effective layer model. b) Spring boundary model.

\section{LINEAR ANGLE BEAM ULTRASONIC SPECTROSCOPY (ABUS) METHOD}

In the linear ABUS method [1, 3-6], pulse-echo normal and pitch-catch oblique incidence signals are recorded simultaneously at each measurement point. The signal from the adhesive layer is then deconvolved by the signal from the reference sample. Bondline properties are extracted from the experimental frequency spectrum using inversion of the direct ultrasonic wave propagation model in composite bonded structures.

The effective layer model was used by us in the past to evaluate degradation in composite bonds (Figure 1a) where the adhesive layer and interface are replaced by an effective layer with effective layer properties [6]. Thus, this model only reveals the combined interfacial/adhesive degradation in composite bonds. In the current work, the spring boundary model [5] used before for aluminum bonds is implemented for composite substrates (Figure 1b). The degraded interface is represented by a decreasing normal or oblique interfacial spring density $K_{\mathrm{n}}$ and $K_{\mathrm{t}}$. The objective is to determine interfacial properties separately from bulk properties of adhesives. The recursive asymptotic stiffness matrix method [7] is integrated into this model to calculate wave propagation in composite bonded structures.
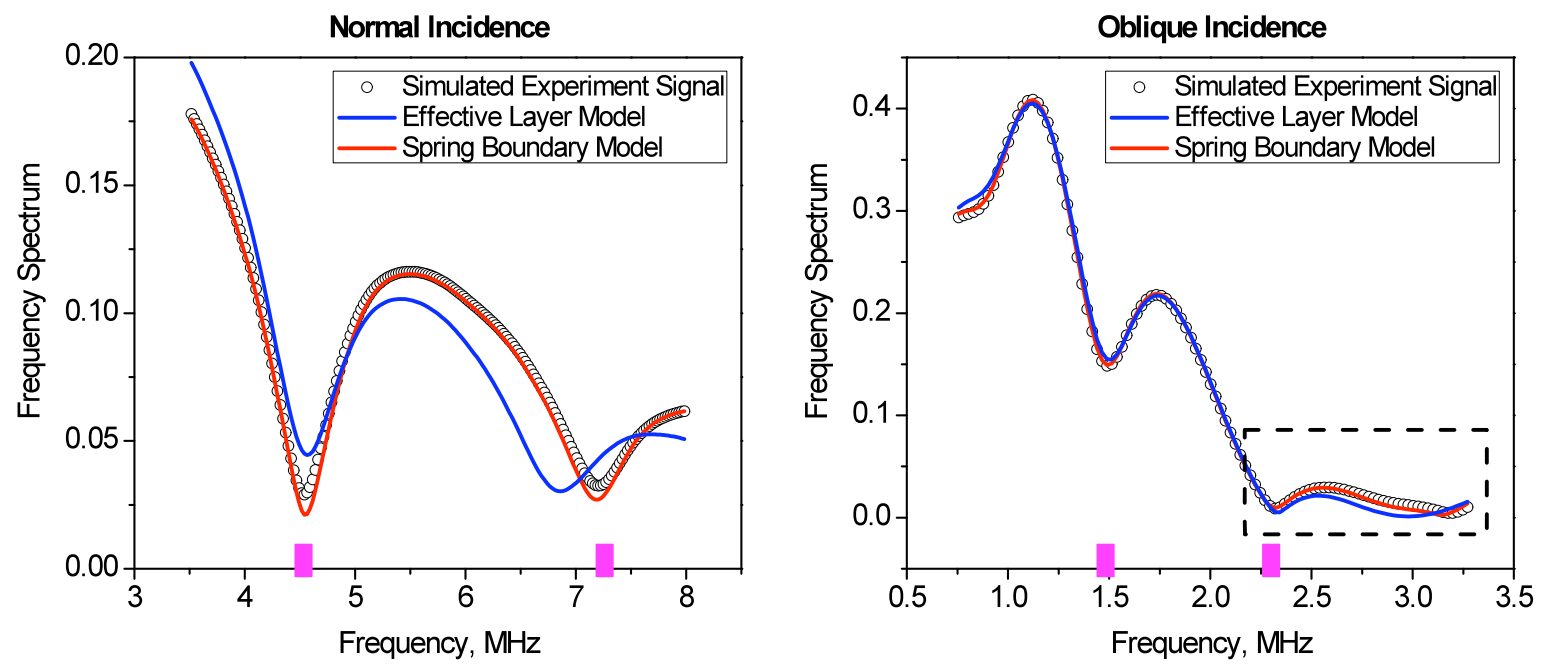

FIGURE 2. Comparison of normal and oblique reflection spectra from composite bond with spectra obtained using reconstruction properties by effective layer and spring boundary ultrasonic measurement models (Fig. 1). 
In Figure 2, the inversion results using both models are compared. The composite substrates considered here are from graphite-epoxy composite with $[0 / 90]_{7 \mathrm{~s}}$ lay-up. At normal incidence, it is possible to observe two resonant frequency dips at $4.5 \mathrm{MHz}$ and $7.25 \mathrm{MHz}$. Thus interfacial properties could be separated from adhesive properties, and the reconstructed normal spring density $K_{\mathrm{n}}$ is reliable (the necessity for two resonance dips in measurements was discussed in [5]). The effective layer model is able to describe well only the first resonant frequency dip; however, with the spring boundary model both resonant frequency dips can be represented well. For the oblique incidence spectrum only one dip is observable due to high distortion/attenuation of the wave spectra at frequencies above $3 \mathrm{MHz}$ [8]. Thus, in such an approach both models are equivalent and it is not possible to separate the effect of interfacial properties from adhesive layer properties. Further enhancement of this approach is needed to address the determination of the transverse spring constant.

\section{MODULATED ANGLE BEAM ULTRASONIC SPECTROSCOPY (ABUS) METHOD}

The sensitivity of the linear ABUS inversion algorithm to interfacial properties is reduced at high interfacial spring densities $K_{\mathrm{n}}$ and $K_{\mathrm{t}}$ (i.e. the method is not sensitive to small initial degradation). Modulated ABUS [2] enhances sensitivity and improves detectability at small interface degradation. In this paper, this method is improved in two ways. First, the measurement threshold is decreased significantly. Second, specimen vibration amplitude is measured ultrasonically at every scanning point. This allows us to locally measure absolute interfacial spring density.

Figure 3 shows the concept of modulated ABUS method which incorporates highfrequency pulsed angle-beam ultrasonic measurements modulated by low-frequency vibration of a bonded structure. It uses parametric/nonlinear mixing between high and low frequencies to characterize the bond line degradation. The low frequency modulation force applied to the bonded sample changes the interface properties and the interfacial spring density. This change
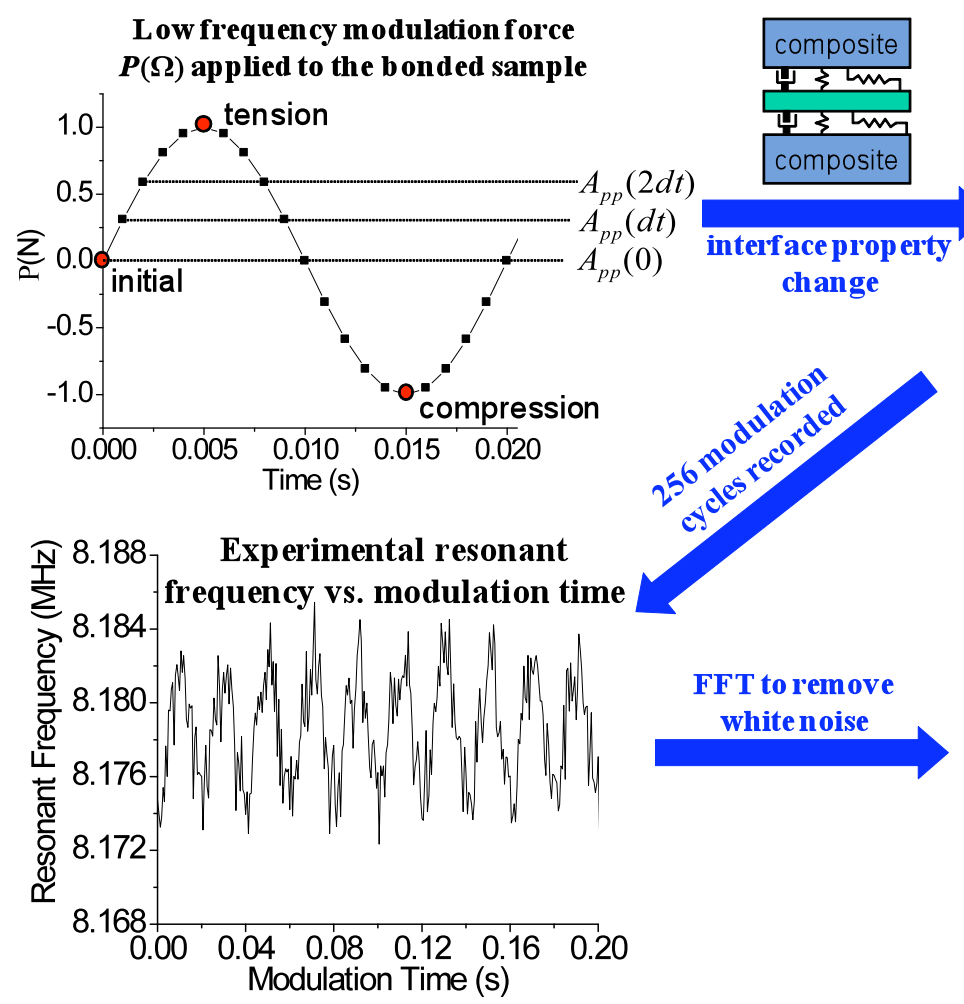
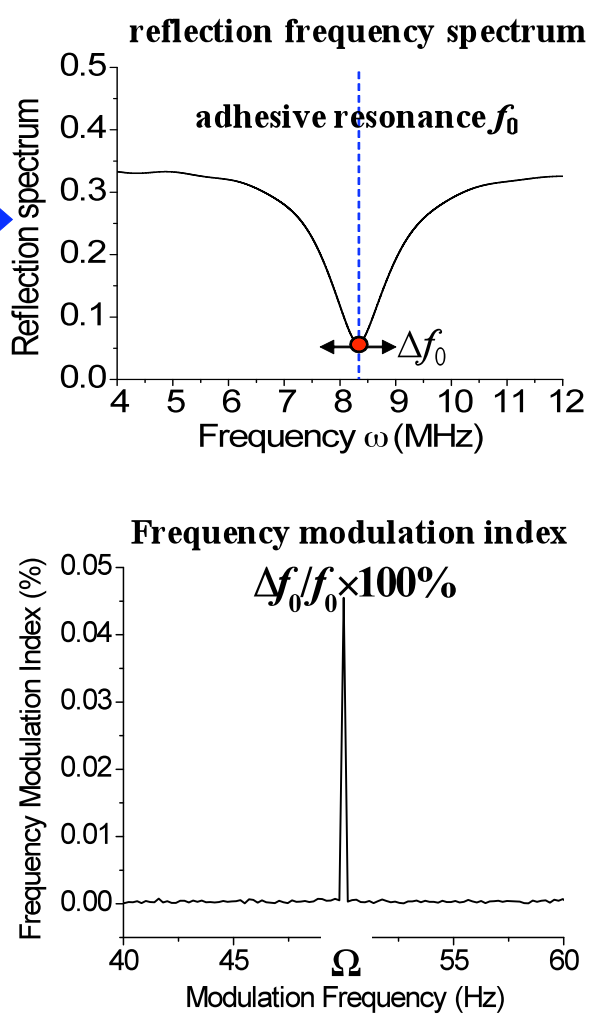

FIGURE 3. The concept of the modulated ABUS method 
results in frequency modulation of the adhesive layer resonance frequency (the reflection spectra dip) which shifts periodically in response to the applied modulation force. In our experiments, 32 ultrasonic pulses per modulation cycle times 256 modulation cycles are recorded for each measured bond line location. The fast Fourier transform (FFT) is applied to remove white noise, and to obtain the frequency modulation index $\mathrm{d} f_{0} / f_{0}$ at the modulation frequency $\Omega$. The degraded interface is associated with a decrease of interfacial spring density and an increase of the frequency modulation index.

\section{Measurement Threshold}

Our objective is to decouple a direct, parasitic, leakage of modulation vibration to the ultrasonic transducer (only modulation due to parametric change of the interface is of interest). We have been able to significantly reduce the signal-to-noise level by improving mechanical damping of the experimental system. Also significant improvement of the signal-to-noise ratio was achieved by increasing the recorded modulation periods, compensating for the effect of low frequency vibration on the recorded signal and parabolic interpolation of the digitized signal to more accurately determine the resonance frequency $f_{0}$ of the adhesive bond to allow accurate determination of the interfacial springs. As a result the modulation frequency signalto-noise ratio threshold is reduced to $0.0004 \%$, which corresponds to $33 \mathrm{~Hz}$ resonance frequency shift (modulation amplitude). This is a significant improvement compared to a previously achieved level of $0.01 \%$ [2]. Our modulation vibration induces bending and pealing loading resulting in complicated displacements in the adhesive bond. Analysis shows that at the current vibration load, the frequency modulation index induced by adhesive layer thickness change is still at least one order of magnitude below the noise level. Thus, frequency modulation can be attributed to interface degradation only.

The improved measurement threshold allows one to obtain small variations resulting from initial environmental degradation of the bonded sample. Figure 4 shows two $2 \mathrm{~cm} \times 2 \mathrm{~cm}$ regions of the bonded specimen (A and B) with different interface qualities. In area A a small level of degradation (small frequency modulation index) is measured which requires high signal-to-noise ratio. In the image A a modulation index variation is significantly above noise level and is well reproducible for repeatable scans without spatial averaging; the image variation is attributed to the bonded interface degradation. In the region B much stronger interface degradation is observed especially at the bottom of the figure closer to the sample edge (note the 4 times larger scale in the frequency modulation index).
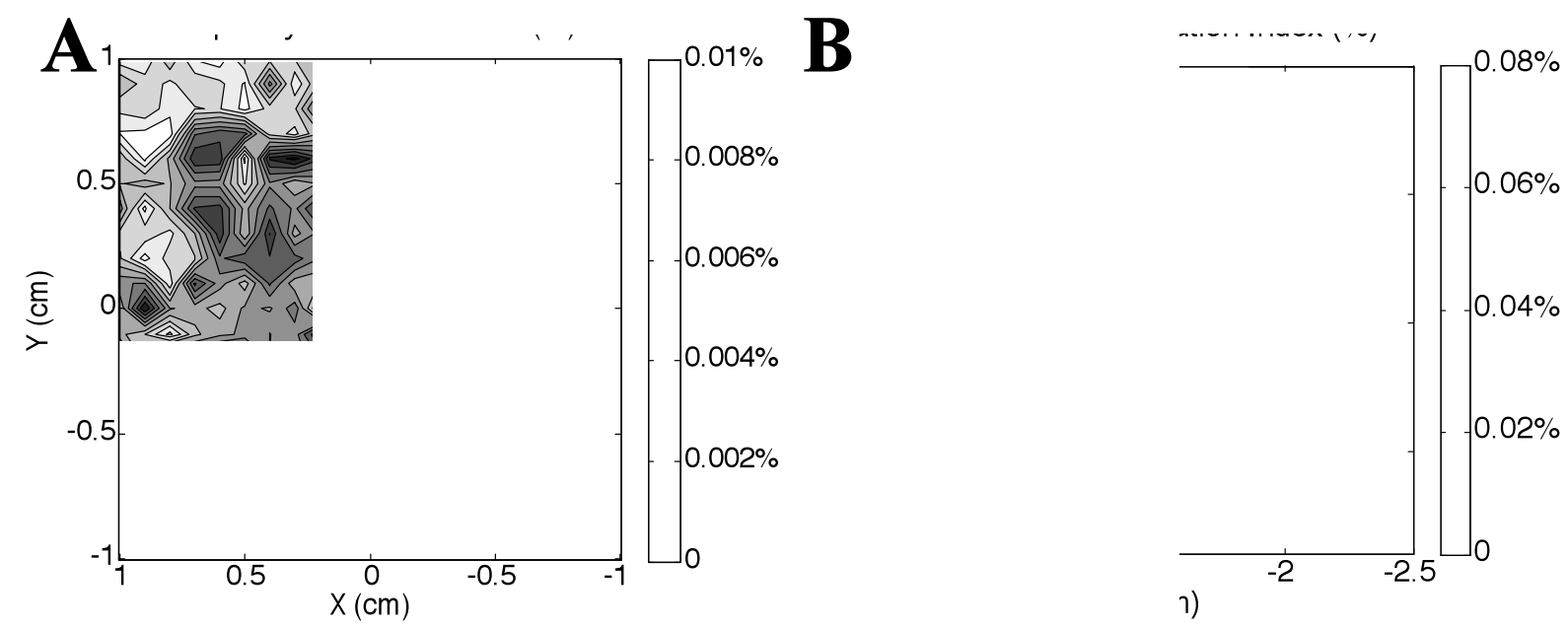

FIGURE 4. An example for Al bond: frequency modulation index 

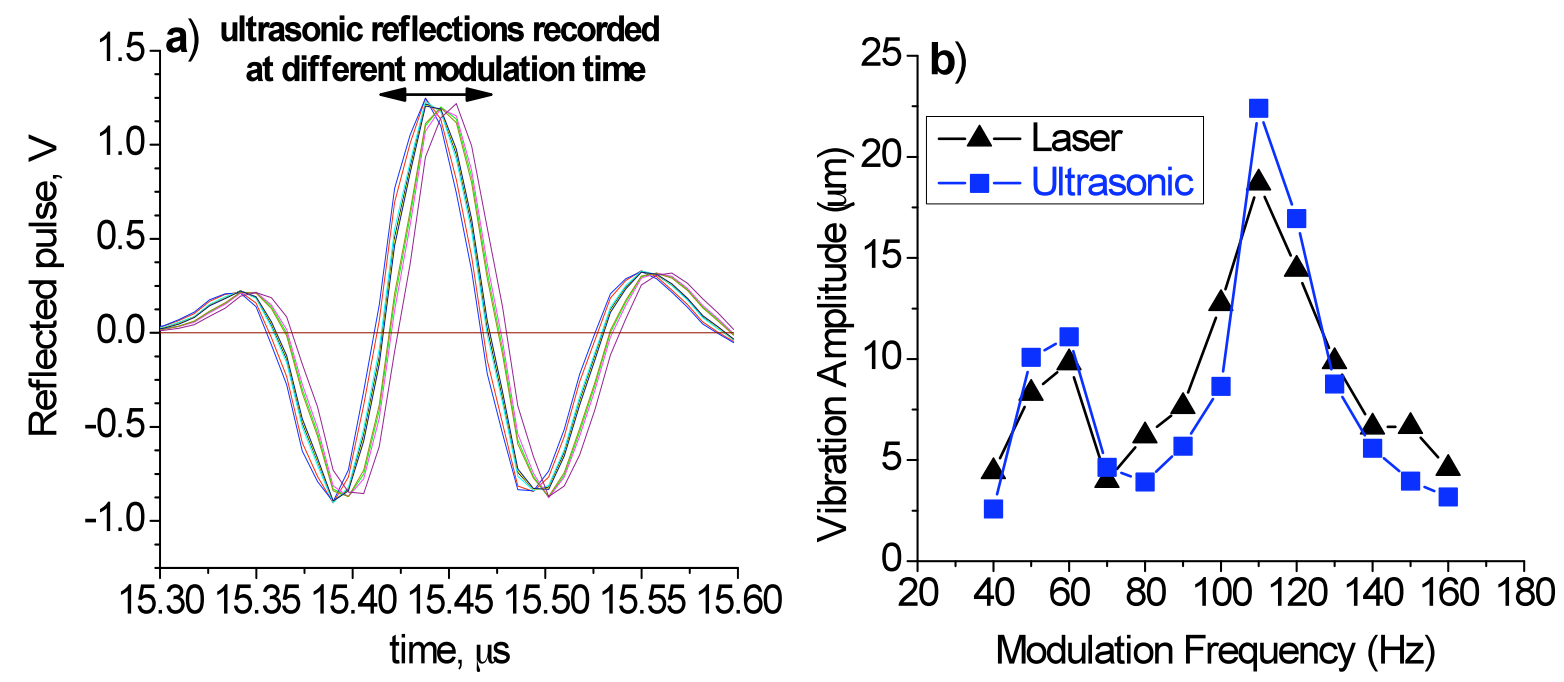

FIGURE 5. a) Ultrasonic measurement of plate vibration amplitude. b) Comparisons of plate vibration amplitude measured by ultrasonic and laser vibrometer methods.

\section{Ultrasonic Measurement of Modulation Vibration Amplitude of the Sample}

To quantitatively relate the measured frequency modulation index to the interface springs knowledge of the modulation vibration amplitude in the given point of the bonded structure is necessary. In this work the sample vibration amplitude in the scanning point is measured ultrasonically simultaneously with the modulation frequency index measurement. As illustrated in Figure 5a, it is obtained by measuring a time-shift of ultrasonic pulses at different modulation time, and converting the time-shift to sample displacement at this scanning point. This signal is filtered from white background noise produced by electronic jitter. Our analysis shows that the measurable displacement threshold is about $100 \mathrm{~nm}$. In Figure $5 \mathrm{~b}$, vibration amplitude measured by this method is compared with absolute displacement measurements using a Polytec ${ }^{(}$laser vibrometer over a modulation frequency range. It is clear that both methods give very similar results.

\section{THE STATISTICAL PHYSICO-CHEMICAL MODEL}

Figure 6 summarizes different approaches to relate ultrasonically measured interfacial spring densities $K_{\mathrm{n}}, K_{\mathrm{t}}$ and frequency modulation index d $f_{0} / f_{0}$ to interface degradation physical models. One is to model the interface degradation as an array of circular cracks [9]. With degradation progress, either the number of cracks or their sizes increase. This model works well for the middle and late stages of degradation when interfacial micro-disbonds have been formed. With the increased sensitivity of the modulation ABUS method the initial degradation can be detected which it is advantageous to describe as distributed damage induced by loss of molecular bonds occurring over the interface area. A physico-chemical model of aluminumepoxy interfaces degradation was developed by Cantrell [10] and it has been recently extended to composite interfaces [11]. In his model, the interface was treated as an array of molecular bonds which are calculated from hydrogen bonding theory. The interface degradation is quantitatively described by decrease of molecular bond density which was related to interfacial spring density $K_{\mathrm{n}}$ and $K_{\mathrm{t}}$ measured ultrasonically. In this work we have extended Cantrell's model by taking into account a statistical distribution of bond interaction length and relating it to the frequency modulation index $\mathrm{d} f_{0} / f_{0}$. We call this new model the statistical physicochemical model. 


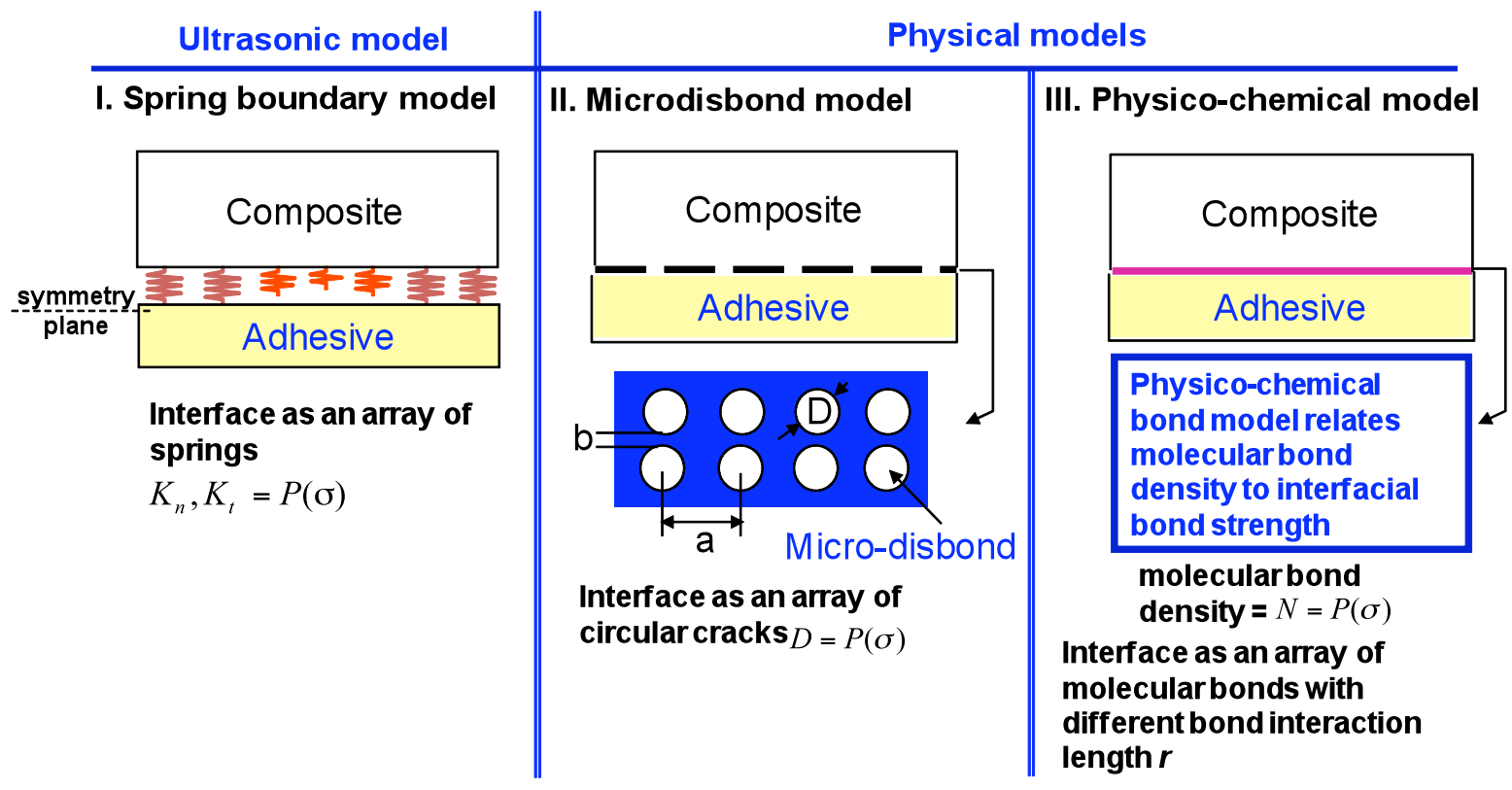

FIGURE 6. Different approaches to relate ultrasonic measurement models with physical models.

There are two components in the statistical physico-chemical model: the chemical bonding model (Figure 7a) and the bond degradation model (Figure 7b). The chemical bonding model defines for a single bond molecular force $T$ as a function of bond interaction length $r$. The relation depends on adhesive and substrate materials, bond type, surface preparation, or temperature. At any moment, the interface contains an array of molecular bonds with different interaction length $r$ and different molecular force $T$. The fraction of molecular bonds $N / N_{0}$ at bond interaction length $r$ is assumed to follow the Weibull distribution:

$$
\frac{N}{N_{0}} \sim W(r ; \delta)=\frac{\pi}{2} \frac{r}{\delta^{2}} e^{-\frac{\pi}{4} \frac{r^{2}}{\delta^{2}}},<r>=\int_{0}^{\infty} r W(r, \delta) d r=\delta
$$

The Weibull distribution is widely applied in describing structural strength distribution at failure [12], especially for materials with many parallel linked elements, like molecular bonds on the interface. The expected value of the Weibull distribution $\delta$ has the physical meaning of the local interface separation. Figure $7 \mathrm{~b}$ indicates that, when degradation proceeds, the local interface separation $\delta$ increases, and the Weibull distribution becomes flat and shifts to higher bond interaction length. For any molecular bond with an interaction length greater than $\delta_{\mathrm{s}}$, this bond is considered completely fractured. The fraction of remaining molecular bonds $N_{r} / N_{0}$ is then given by integration from 0 to $\delta_{\mathrm{s}}$ as

$$
\frac{N_{r}}{N_{0}}=\int_{0}^{\delta_{s}} W(r, \delta) d r
$$

Combining the chemical bonding model for a single bond (Figure 7a) with the bond degradation model (Figure 7b), we obtain the interface binding force $\sigma$ as a function of local interface separation $\delta$

$$
\sigma(\delta)=\int_{0}^{\infty} T(r) W(r, \delta) d r
$$

As shown in Figure 7c, when damage is not initiated, the interface has the maximum binding force $\sigma_{0}$ at initial local interface separation $\delta_{0}$. On the degraded interface A, modulation load changes boundary conditions of the interface and moves the binding force between $\mathrm{B}$ and $\mathrm{C}$. At each modulation point between $\mathrm{B}$ and $\mathrm{C}$, ultrasonic signals measure interface stiffness, and its slope is the interfacial spring density $k$. Different interfacial spring density $k$ has different resonant frequency $f_{0}$, and causes experimental frequency modulation index $\mathrm{d} f_{0} / f_{0}$. 

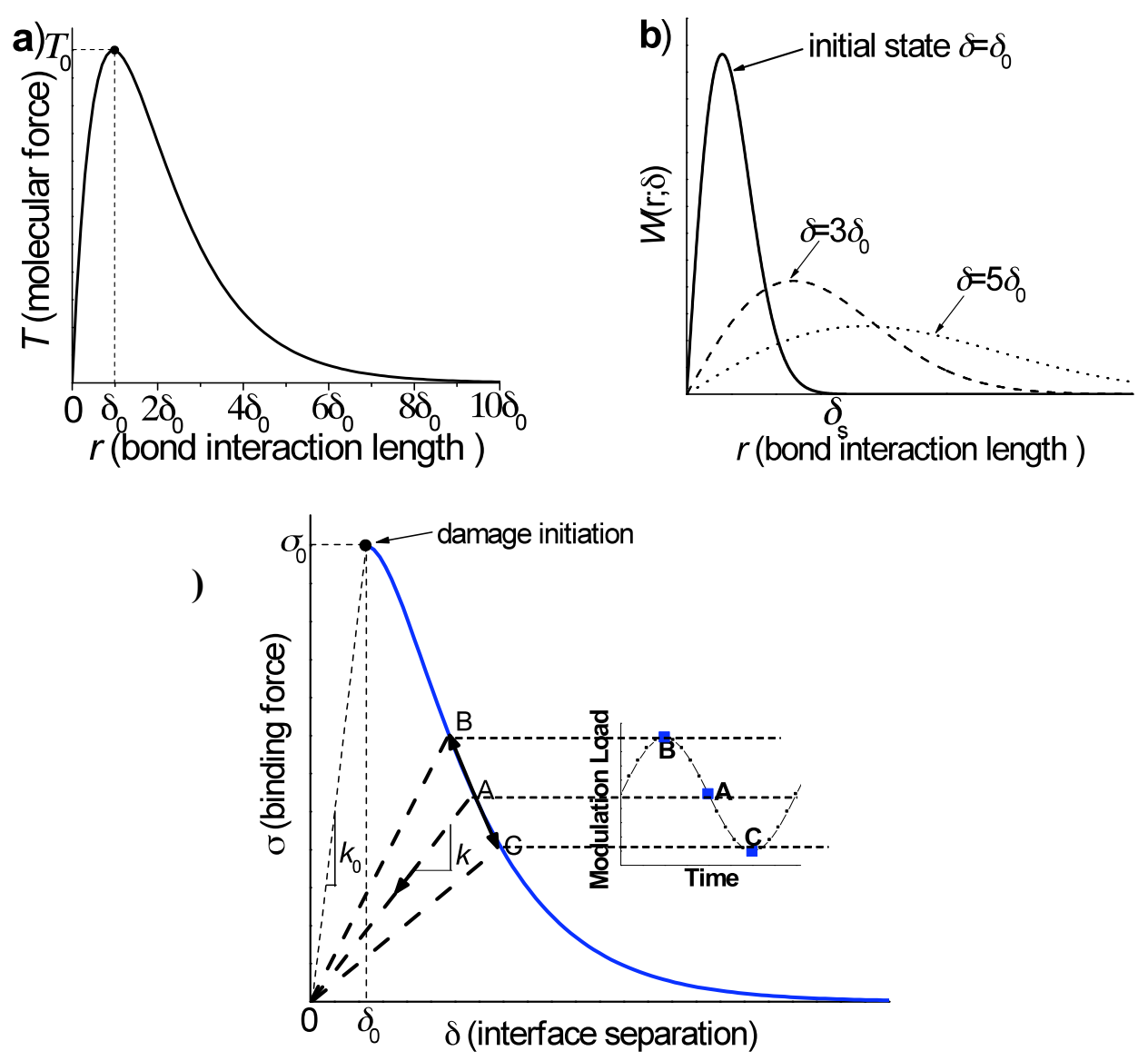

FIGURE 7. Elements of the statistical physico-chemical model.

Different physico-chemical bonding mechanisms are modeled by different molecular potentials (Morse potential Eq. (4) [12]; Lennard-Jones potential used to model the van der Waals forces Eq. (5); Rose-Ferrante-Smith universal binding energy Eq. (6) [13]):

$$
\begin{gathered}
U=U_{0}\left(e^{-2 a\left(r-r_{0}\right)}-2 e^{-a\left(r-r_{0}\right)}\right), \quad T=-\frac{\partial U}{\partial r} \\
U=-4 \varepsilon\left[\left(\frac{\sigma}{r}\right)^{6}-\left(\frac{\sigma}{r}\right)^{12}\right], \quad T=-\frac{\partial U}{\partial r} \\
U=-e T_{0} \delta_{0}\left(1+\frac{r}{\delta_{0}}\right) e^{-\frac{r}{\delta_{0}}}, \quad T=-\frac{\partial U}{\partial r}
\end{gathered}
$$

Figure 8 shows an application example of the statistical physico-chemical model using Rose-Ferrante-Smith universal binding energy, where the measured frequency modulation index $\mathrm{d} f_{0} / f_{0}$ is related to interfacial spring density and loss of molecular bonds. To calculate this, the maximum binding force $\sigma_{0}$ is taken as $30 \mathrm{MPa}$, typical of adhesive bond strength. The integrated area under Figure $7 \mathrm{c}$ is taken as $1 \mathrm{~J} / \mathrm{m}^{2}$ which is typical for the interfacial surface energy density. The modulation load in the specimen is estimated as $3.4 \mathrm{KPa}$. The frequency modulation index $\mathrm{d} f_{0} / f_{0}$ increases 100 times from $0.0003 \%$ at nearly perfect bonding to $0.03 \%$ at $80 \%$ loss of molecular bonds. It is similar to the range of frequency modulation index measured in our experiments (Fig. 4). At the initial degradation, the decrease of normal interfacial spring density $K_{n}$ is proportional to the increase of the frequency modulation index $\mathrm{d} f_{0} / f_{0}$. The measurement threshold of the frequency modulation index of $0.0004 \%$ corresponds to a $1.4 \%$ loss of molecular bond per unit area (fraction of disbonded area) or a value of normal interfacial spring density as high as $2 \times 10^{15} \mathrm{~N} / \mathrm{m}^{3}$. 


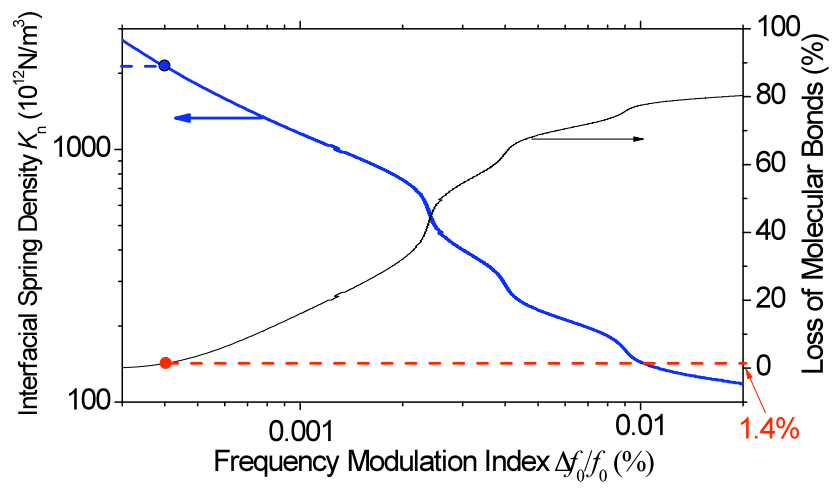

FIGURE 8. An application example of the statistical physico-chemical model.

\section{SUMMARY}

In this paper, linear and nonlinear (modulated) angle beam ultrasonic spectroscopies have been applied to separate interfacial and adhesive properties from ultrasonic measurements of composite bonds. For early detection of interface degradation in the bond significant improvement of signal-to-noise ratio was obtained in the modulated method. The threshold of $0.0004 \%$ in the frequency modulation index was achieved, which corresponds to a sensitivity of $1.4 \%$ in measurement of the loss of molecular bonds per unit area. A statistical physicochemical model of bonded interface degradation is developed to relate measured frequency modulation index to loss of interfacial molecular bond density.

\section{ACKNOWLEDGEMENTS}

This work is sponsored by NASA Grant No. NNX08BA37A.

\section{REFERENCES}

1. A. I. Lavrentyev and S. I. Rokhlin, J. Acoust. Soc. Am. 102, 3467-3477 (1997).

2. S. I. Rokhlin, L. Wang, B. Xie, V. A. Yakovlev and L. Adler, Ultrasonics 42, 1037-1047 (2004).

3. S.I. Rokhlin, B. Xie and A. Baltazar, J. Adhesion Sci. Tech., 18 (3) 327-360 (2004).

4. L. Wang, B. Xie and S. I. Rokhlin, J. Acoust. Soc. Am. 111, 2644-2653 (2002).

5. A. Baltazar, L. Wang, B. Xie and S. I. Rokhlin, J. Acoust. Sos. Am. 114, 1424-1434 (2003).

6. L. Adler, S. I. Rokhlin, C. Mattei, G. Blaho, and Q. Xie, Review of Progress in QNDE, 18B, Eds. D. O. Thompson and D. E. Chimenti, Plenum, 1999, pp. 1553-1559.

7. L. Wang and S. I. Rokhlin, J. Mech. Phys. Solids 52, 2473-2506 (2004).

8. L. Wang and S. I. Rokhlin, J. Acoust. Sos. Am. 114, 2582-2595 (2003).

9. A. I. Lavrentyev and S. I. Rokhlin, J. Appl. Phys. 76, 4643-4650 (1994).

10. J. H. Cantrell, J. Appl. Phys. 96, 3775-3781 (2004).

11. J. H. Cantrell, "Hydrogen bonds, interfacial stiffness moduli, and the interlaminar shear strength of carbon fiber-epoxy matrix composites", (unpublished).

12. Z. P. Bažant, S.-D. Pang, J. Mech. Phys. Solids 55, 91-131 (2007).

13. J. H. Rose, J. Ferrante and J. R. Smith, Phys. Rev. Lett. 42, 675-678 (1981). 